\title{
Editorial: Employing Experimental Gnotobiotic Models to Decipher the Host-Microbiota Cross-Talk in Health and Disease
}

\author{
Martin Schwarzer ${ }^{1 *}$, Helena Tlaskalova-Hogenova ${ }^{2}$, François Leulier ${ }^{3}$ \\ and Irma Schabussova ${ }^{4}$ \\ 1 Laboratory of Gnotobiology, Institute of Microbiology, Czech Academy of Sciences, Novy Hradek, Czechia, ${ }^{2}$ Laboratory of \\ Cellular and Molecular Immunology, Czech Academy of Sciences, Prague, Czechia, ${ }^{3}$ Institut de Génomique Fonctionnelle de \\ Lyon, Université de Lyon, Ecole Normale Supérieure de Lyon, Centre National de la Recherche Scientifique, Université \\ Claude Bernard Lyon 1, Unité Mixte de Recherche 5242, Lyon, France, ${ }^{4}$ Institute of Specific Prophylaxis and Tropical \\ Medicine, Medical University of Vienna, Vienna, Austria
}

Keywords: germ-free, host-bacteria interactions, microbiota, gnotobiology, immune system

Editorial on the Research Topic

Employing Experimental Gnotobiotic Models to Decipher the Host-Microbiota Cross-Talk in Health and Disease

\section{OPEN ACCESS}

Edited and reviewed by: Nils Yngve Lycke, University of Gothenburg, Sweden

${ }^{*}$ Correspondence: Martin Schwarzer schwarzer@biomed.cas.cz

Specialty section:

This article was submitted to

Mucosal Immunity,

a section of the journal

Frontiers in Immunology

Received: 22 June 2021

Accepted: 19 July 2021

Published: 03 August 2021

Citation:

Schwarzer M,

Tlaskalova-Hogenova $\mathrm{H}$,

Leulier F and Schabussova I (2021) Editorial: Employing Experimental Gnotobiotic Models to Decipher the Host-Microbiota Cross-Talk

in Health and Disease.

Front. Immunol. 12:729052. doi: 10.3389/fimmu.2021.729052

\section{INTRODUCTION}

Eukaryotic organisms have evolved in a world dominated by bacteria and archaea. Rather than face the daunting task of keeping their exposed surfaces germ-free (GF), they have developed close symbiotic relationships. Mucosal surfaces are associated with specific microbial communities that influence various aspects of host physiology and, most importantly, the development and finetuning of the immune system. In the last decade, we have witnessed a renewed interest in understanding the role of the microbiota for the homeostasis and disease. This has fostered the development of sophisticated multidisciplinary technologies that enable compositional and functional analysis of the microbiome (1). The most diverse and numerous microbial communities are found in the gastrointestinal tract. Alterations in the gut microbiome and/or disruptions of the cross-talk between host and microbiota has been linked to immune-mediated diseases such as allergies and autoimmune diseases such as inflammatory bowel disease, rheumatoid arthritis, multiple sclerosis and diabetes (2). The aforementioned complexity of the gut microbial ecosystem currently complicates the understanding of the microbiota-host cross-talk, with descriptive reports predominating over mechanistic studies. However, powerful tools for studying host-microbe interactions are germ-free (GF) and gnotobiotic animal models. Although historically mostly rodents and piglets (3) have been used, new vertebrate models, for example fish, and invertabrate models such as Drosophila have successfully been developed in recent years (4).

The possibility to colonize GF animals with defined bacterial species or a consortium of bacteria has created a unique opportunity to evaluate the impact of the microbiota on host physiology and immune system (5). Colonization of GF mice with a defined bacterial consortium that mimics the complexity of a specific pathogen-free/specific opportunistic pathogen-free (SPF/SOPF) microbiota has increased the reproducibility of mouse experiments across different institutions and mouse 
facilities (6). In this regard, Eberl et al. show that one such consortium, Oligo-Mouse-Microbiota12 (Oligo-MM12), could be established in several different animal facilities with excellent reproducibility. Within two weeks after inoculation of the OligoMM12 consortium in GF mice similar stable microbial communities were found in all facilities. Furthermore, they showed that a second inoculation of the Oligo-MM12 strains after $72 \mathrm{~h}$ was even more effective at establishing a stable microbiota. Thus the reliable de novo generation of gnotobiotic rodents contributes greatly to experimental reproducibility, which has significantly benefitted biomedical research in recent years. Using the Oligo-MM12 consortium, Wyss et al. sought to explain the phenomenon behind elevated IgE levels in GF animals. Depending on the mouse facility, mouse model, and breeding conditions, GF mice exhibit elevated total serum IgE levels (6-9). Wyss et al. demonstrated that colonization of mice with a well-defined composition of bacterial species is required to inhibit elevated IgE levels. Features of bacterial consortia that successfully decrease the IgE include presence in early life, acetate production, and immunogenicity reflected in the induction of gut intestinal IgA.

There is a growing interest in the development of microbialbased and microbial-targeted therapies (10). Preclinical studies with "humanized" mice (transfer of human microbiota to germfree animals) are often necessary methodological requirement to analyze the potential therapeutic effects. This topic was discussed in detail by Rogala et al. GF mice colonized with single wild-type or genetically engineered microbial isolates are an invaluable tool to study the functions of individual bacterial genes and species. GF mice colonized with multiple defined isolates can be used to determine interactions between members of defined consortia. This is elegantly discussed and ways to improve studies of immune-microbial interactions using gnotobiotic mice are presented.

On this theme, Bolsega et al. investigated whether different bacterial minimal consortia affect the outcome of murine norovirus-induced colitis in $1110^{-/-}$mice. By comparing two different minimal microbiota (Oligo-MM12 and Altered Schaedler Flora) colonized mice, they show that murine norovirus-triggered colitis depends on the composition of the microbiota. Lengfelder et al. characterized the colitogenic activity of Enterococcus faecalis as part of a simplified human microbial consortium based on seven enteric bacterial strains (SIHUMI). They showed that complex bacterial consortium interactions reprogram the gene expression profile and colitogenic activity of the opportunistic pathogen E. faecalis towards a protective

\section{REFERENCES}

1. Heintz-Buschart A, Wilmes P. Human Gut Microbiome: Function Matters. Trends Microbiol (2018) 26:563-74. doi: 10.1016/j.tim.2017.11.002

2. Tlaskalova-Hogenova H, Stepankova R, Kozakova H, Hudcovic T, Vannucci L, Tuckova L, et al. The Role of Gut Microbiota (Commensal Bacteria) and the Mucosal Barrier in the Pathogenesis of Inflammatory and Autoimmune Diseases and Cancer: Contribution of Germ-Free and Gnotobiotic Animal Models of Human Diseases. Cell Mol Immunol (2011) 8:110-20. doi: 10.1038/ cmi.2010.67 function. Kostovcikova et al. examined colitis from a nutritional perspective and used a gnotobiotic approach to show that diet rich in animal protein exacerbates acute dextran sulfate sodium (DSS)-induced colitis, whereas diet rich in plant protein does not. They concluded that interactions between a dietary protein of animal origin and the gut microbiota increase sensitivity to intestinal inflammation by promoting the proinflammatory responses of monocytes.

The gut microbiota can influence brain functions and behavior, including hypothalamic-pituitary-adrenocortical axis (HPA) activity. Using SPF and GF male BALB/c mice, Vagnerova et al. investigated the influence of the microbiota on the acute restraint stress (ARS) response in the pituitary, adrenal gland, and intestine, an organ of extra-adrenal glucocorticoid synthesis. They showed that GF animals have an exaggerated HPA response to stress.

Finally, Murdoch and Rawls review the key insights provided by the gnotobiotic zebrafish model on the effects of microbiota on innate immunity. This included evidence that the perception of and response to the microbiota is evolutionarily conserved. They describe how to strengthen the zebrafish model system and provide new insights into the host-microbe interactions that would be difficult to study in mammalian models.

Overall, we have assembled a set of eight research articles that bring new insights into host-microbiota interactions. These studies all highlight the advantages of gnotobiotic rodent and fish models, and demonstrate the modularity of defined bacterial minimal consortia that can be used to achieve greater standardization of biomedical research across different animal facilities.

\section{AUTHOR CONTRIBUTIONS}

All authors listed have made a substantial, direct and intellectual contribution to the work, and approved it for publication.

\section{FUNDING}

This work was supported by grant $18-07015 Y$ from the Czech Science Foundation and EMBO Installation grant to MS, The Danube-ARC funded by the Government of Lower Austria to IS and Czech Health Research Council grant NV19-03-00179 to HT-H.

3. Tlaskalova-Hogenova H, Sterzl J, Stepankova R, Dlabac V, Veticka V Rossmann P, et al. Development of Immunological Capacity Under Germfree and Conventional Conditions. Ann N Y Acad Sci (1983) 409:96113. doi: 10.1111/j.1749-6632.1983.tb26862.x

4. Leulier F, MacNeil LT, Lee WJ, Rawls JF, Cani PD, Schwarzer M, et al. Integrative Physiology: At the Crossroads of Nutrition, Microbiota, Animal Physiology, and Human Health. Cell Metab (2017) 25:522-34. doi: 10.1016/ j.cmet.2017.02.001

5. Macpherson AJ, McCoy KD. Independence Day for IgA. Immunity (2015) 43:416-8. doi: 10.1016/j.immuni.2015.08.024 
6. Darnaud M, Vadder FD, Bogeat P, Boucinha L, Bulteau A-L, Bunescu A, et al. A Standardized Gnotobiotic Mouse Model Harboring a Minimal 15-Member Mouse Gut Microbiota Recapitulates SOPF/SPF Phenotypes. bioRxiv (2021). doi: 10.1101/2019.12.30.890954

7. Cahenzli J, Köller Y, Wyss M, Geuking MB, McCoy KD. Intestinal Microbial Diversity During Early-Life Colonization Shapes Long-Term IgE Levels. Cell Host Microbe (2013) 14:559-70. doi: 10.1016/j.chom.2013.10.004

8. Hong SW, O E, Lee JY, Lee M, Han D, Ko HJ, et al. Food Antigens Drive Spontaneous IgE Elevation in the Absence of Commensal Microbiota. Sci Adv (2019) 5:eaaw1507. doi: 10.1126/sciadv.aaw1507

9. Rodriguez B, Prioult G, Bibiloni R, Nicolis I, Mercenier A, Butel M-J, et al. Germ-Free Status and Altered Caecal Subdominant Microbiota Are Associated With a High Susceptibility to Cow's Milk Allergy in Mice. FEMS Microbiol Ecol (2011) 76:133-44. doi: 10.1111/j.1574-6941.2010. 01035.x

10. Oka A, Sartor RB. Microbial-Based and Microbial-Targeted Therapies for Inflammatory Bowel Diseases. Dig Dis Sci (2020) 65:757-88. doi: 10.1007/ s10620-020-06090-Z
Conflict of Interest: The authors declare that the research was conducted in the absence of any commercial or financial relationships that could be construed as a potential conflict of interest.

Publisher's Note: All claims expressed in this article are solely those of the authors and do not necessarily represent those of their affiliated organizations, or those of the publisher, the editors and the reviewers. Any product that may be evaluated in this article, or claim that may be made by its manufacturer, is not guaranteed or endorsed by the publisher.

Copyright (C) 2021 Schwarzer, Tlaskalova-Hogenova, Leulier and Schabussova. This is an open-access article distributed under the terms of the Creative Commons Attribution License (CC BY). The use, distribution or reproduction in other forums is permitted, provided the original author(s) and the copyright owner(s) are credited and that the original publication in this journal is cited, in accordance with accepted academic practice. No use, distribution or reproduction is permitted which does not comply with these terms. 